ISSN 1518-3483

Licenciado sob uma Licença Creative Commons

(c) (i)

\title{
Experiência de Educação Ambiental utilizando Pesquisa e Inovação Responsáveis da Pontifícia Universidade Católica do Paraná no Projeto Europeu Engage
}

Experience of Environmental Education using Responsible Research and Innovation of the Pontifical Catholic University of Paraná in the European Project Engage

Patrícia Lupion Torres ${ }^{[a]}$, Katia Ethiénne Esteves dos Santos ${ }^{[a][b]}$, Raquel Pasternak Glitz Kowalski ${ }^{[\mathrm{la}[\mathrm{cc}]}$, Alexandra Okada ${ }^{[\mathrm{d}]]^{*}}$

\footnotetext{
[a] Pontifícia Universidade Católica do Paraná (PUCPR), Curitiba, PR, Brasil

[b] Universidade Positivo, Curitiba, PR, Brasil

[c] FAE Centro Universitário, Curitiba, PR, Brasil

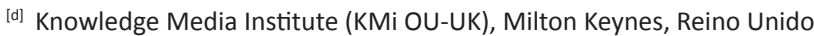

\section{Resumo}

Este estudo investiga como o grupo de pesquisa Prática Pedagógica na Educação Presencial e a Distância: Metodologias e Recursos Inovadores de Aprendizagem PRAPETEC - da Pontifícia Universidade Católica do Paraná (PUCPR), em parceria com o projeto europeu

PLT: Doutora em Engenharia de Produção, e-mail: patorres@terra.com.br KEES: Doutoranda em Educação, e-mail: katiaethienne@uol.com.br RPGK: Doutoranda em Educação e-mail: raquel.pasternak@pucpr.br AO: Doutora em Educação, e-mail: alexandra.okada@gmail.com 
Engage, promoveu atividades com estudantes do Ensino Médio e Superior para desenvolver novas estratégias para equipar os estudantes, para aplicar o conhecimento científico e desenvolver habilidades de pesquisa RRI (Responsible Research and Innovation - Pesquisa e Inovação Responsáveis) durante o ano de 2015. O projeto europeu Engage (engagementingscience.eu) tem como objetivo aumentar a conscientização da Pesquisa e Inovação Responsáveis (RRI). A PUCPR, em parceria com o projeto Engage, realizou uma série de atividades, que promoveram a educação ambiental, envolvendo a Agrobiodiversidade - Transgênicos, por meio de Recursos Educacionais Abertos (REA) e a cocriação. Neste contexto, os professores desempenham um papel importante para tornar a educação ambiental mais relevante para as preocupações dos alunos, que são conhecidos por serem orientados para o futuro. Isso pode aumentar a probabilidade de que os alunos possam aplicar o que aprenderam fora da escola e, também, responderem a desafios sociais.

Palavras-chave: REA - Recursos Educacionais Abertos. RRI - Pesquisa e inovação Responsável. Cocriação. Engage.

\section{Abstract}

This study investigates how the research group Pedagogical Practice in Distance Learning: Innovative Methodologies and Learning Resources PRAPETEC - of the Pontifical Catholic University of Paraná (PUCPR) in partnership with the European project Engage, promoted activities with high school students and to develop new strategies for equipping students to apply scientific knowledge and to develop RRI (Responsible Research and Innovation) research skills during the year 2015. The European project Engage (engagementingscience. eu) aims to increase awareness of Responsible Research and Innovation (RRI). PUCPR in partnership with the Engage project carried out a series of activities, which promoted environmental education involving Agrobiodiversity - Transgenics, through Open Educational Resources (OER) and co-creation. In this context, teachers play an important role in making environmental education more relevant to students' concerns, which are known to be forward-looking. This can increase the likelihood that students can apply what they have learned out of school and respond to social challenges. 
Keywords: OER - Open Educational Resources. RRI - Responsible Research and innovation. Cocreation. Engage.

\section{Resumen}

Este estudio investiga como el grupo de investigación Práctica Pedagógica en la Educación Presencial y la Distancia: Metodologías y Recursos Innovadores de Aprendizaje PRAPETEC - de la Pontificia Universidad Católica de Paraná (PUCPR) en asociación con el proyecto europeo Engage, promovió actividades con estudiantes de la Enseñanza Media y, Superior para desarrollar nuevas estrategias para equipar a los estudiantes para aplicar el conocimiento científico y desarrollar habilidades de investigación RRI (Responsable Research and Innovation - Investigación e Innovación Responsables) durante el año 2015. El proyecto europeo Engage (engagementingscience.eu) tiene como objetivo aumentar la concientización de la Investigación e Innovación Responsables (IIR). La PUCPR en asociación con el proyecto Engage realizó una serie de actividades, que promovió la educación ambiental envolviendo la Agrobiodiversidad - Transgénicos, a través de Recursos Educativos Abiertos (REA) y la cocción. En este contexto, los profesores desempeñan un papel importante para hacer que la educación ambiental más relevante para las preocupaciones de los alumnos, que son conocidas por estar orientadas hacia el futuro. Esto puede aumentar la probabilidad de que los alumnos puedan aplicar lo que aprendieron fuera de la escuela y responder a los desafios sociales.

Palabras clave: REA - Recursos Educativos Abiertos. RRI - Investigación e innovación Responsable. Cocción. Engage. 


\section{Introdução}

\section{RRI e o Projeto Engage}

O século XXI é marcado pelo acelerado avanço na tecnologia e na ciência. No cenário contemporâneo, a educação desempenha um papel importante nas transformações socioculturais. A Comissão Europeia destacou a importância da Pesquisa e Inovação Responsáveis (Criando o conceito em inglês: Responsible Research and Innovation - RRI) na educação científica por meio dos seus programas de ciência na sociedade (FP7 e Horizon 2020).

Pesquisa e Inovação Responsáveis é uma abordagem que antecipa e avalia as implicações potenciais e as expectativas da sociedade em matéria de investigação e inovação, com o objetivo de promover uma investigação e inovação inclusivas e sustentáveis (COMISSÃO EUROPEIA, 2012).

Vários projetos europeus têm auxiliado os docentes a promover habilidades de aprendizagem baseada em investigação (Inquiry Based Learning - IBL) para que os estudantes possam discutir questões sociais e científicas considerando a abordagem RRI, como por exemplo o projeto europeu Engage (OKADA et al., 2015).

O projeto ENGAGE, financiado pela Comissão Europeia, visa difundir o ensino e a aprendizagem do RRI, por meio da ciência e tecnologia com aprendizagem baseada em investigação. Um grande desafio para os docentes é transformar a forma como a ciência é ensinada em uma prática de ensino inovadora. Isso requer que o ensino da ciência inclua um conjunto de conteúdos e práticas para propiciar aos estudantes oportunidades para que os seu conhecimentos, habilidades e valores sejam desenvolvidos para usar a ciência no cotidiano.

O currículo para RRI desenvolvido pelo projeto Engage apresenta uma estrutura que integra conhecimentos de ciência e habilidades de investigação científica, com atividades práticas baseadas em dilemas científicos para discussão, argumentação com base de jogos, resolução de 
problemas, desenvolvimento de projetos e avaliação da performance. O conhecimento da ciência é baseado em quatro áreas principais: impacto tecnológico, ciência, pensamento de valores e ciência na mídia:

- Impacto tecnológico: os desenvolvimentos tecnológicos e científicos são a base para um futuro melhor, mas devem ser planejados cuidadosamente para maximizar os benefícios e reduzir riscos.

- Ciência: A ciência não visa apenas uma busca individual por conhecimento, mas um processo colaborativo para beneficiar a sociedade e o mundo complexo. A ciência financiada principalmente por corporações e governos, favorece aplicações práticas na sociedade. Isto significa que inovações responsáveis devem abordar as necessidades da sociedade de acordo com seus valores, tais como sociais, ambientais e econômicos.

- Pensamento de valores: nas ciências e tecnologias emergentes, muitas vezes há situações-problemas incertas, com implicações pouco claras, que requerem um pensamento sócio ético. As decisões devem ser tomadas levando em consideração as opiniões e preocupações de várias perspectivas e atores nas sociedades com base na ética.

- Ciência na mídia: grande parte de nossa informação científica é interpretada pela mídia, que pode dar uma informação desequilibrada, tendenciosa ou sensacionalista. A fonte de informação precisa ser avaliada em termos de sua finalidade, confiabilidade das credenciais científicas e referências. Ciência na mídia é importante para envolver cidadãos na reflexão crítica dos assuntos científicos. Isso requer que indivíduos leiam criticamente informações da mídia sobre ciência, identifiquem os dados ou fatos usados como evidência e valores implícitos.

Okada (2016) destaca que o currículo RRI do projeto Engage destina-se a três componentes: o interesse dos estudantes, conhecimento científico e as 10 habilidades de investigação científica para RRI: 
1) Elaborar perguntas: ser capaz de definir uma questão científica clara para investigar as relações de causa ou correlação entre diferentes fatores.

2) Interrogar fontes: ser capaz de questionar diferentes fontes e avaliar a sua validade e confiabilidade, julgando a qualidade da fonte, verificando o viés e avaliando a evidência de reivindicação.

3) Analisar consequências: ser capaz de avaliar o mérito de uma solução ou soluções concorrentes para um problema do mundo real (incluindo processos ou métodos utilizados), com base em ideias científicas, princípios e evidências empíricas, identificando e refletindo sobre as consequências ou implicações em relação à economia, sociedade e considerações ambientais.

4) Estimar riscos: ser capaz de medir riscos e benefícios avaliando sua probabilidade, ponderando e combinando sua probabilidade e a escala de seu impacto, além de equilibrar os benefícios com os indivíduos ou grupos afetados.

5) Analisar dados: ser capaz de interpretar observações e dados em uma variedade de formas identificando padrões e tendências, fazer inferências e extrair conclusões.

6) Tirar conclusões: ser capaz de decidir se a reivindicação feita por uma pesquisa é suportada por dados suficientes, refletindo na análise e métodos utilizados.

7) Justificar opiniões: ser capaz de sintetizar e conectar o conhecimento científico, implicações e perspectivas de valor em uma opinião informada, com argumentos apoiados por evidências e raciocínio científico com base em valores explícitos, que apoiam ou não um ponto de vista sobre uma questão ou solução para um problema.

8) Criticar afirmações: ser capaz de verificar a força (precisão de qualidade e suficiência) da evidência fornecida e identificar a falta de clareza da justificativa, comentando se o raciocínio segue logicamente da evidência e fornece um forte apoio à reivindicação.

9) Usar a ética: ser capaz de compreender e usar o pensamento ético: por exemplo, utilitarismo, direitos e deveres, virtudes e/ou para 
tomar decisões informadas e explicar por que diferentes pessoas podem ter diferentes pontos de vista sobre um problema.

10) Comunicar ideias: ser capaz de descrever efetivamente opiniões e argumentações com referências e ilustrações, tanto oralmente quanto por escrito, em uma variedade de formatos, usando as principais características da escrita e fala científica.

Para propiciar as habilidades de investigação científica para o RRI na educação, o projeto Engage foi desenhado para facilitar o crescimento do conhecimento conceitual e prático dos docentes utilizando REA e ações presenciais e online por meio das três etapas seguintes: adotar, adaptar e transformar (AIKENHEAD, 1994).

Estes três estágios indicam um processo por etapas para que professores possam gradualmente inovar o currículo existente considerando o conteúdo da ciência e da sociedade que já está integrado em suas aulas com novos conteúdos e práticas da ciência contemporânea da RRI:

a) Etapa 1 - Adotar: fase inicial com pequenas mudanças — o papel do professor é estender tópicos já ensinados com lições curtas sobre dilemas sócio científicos e discussão em grupo do Engage. Esta fase apresenta pouco conteúdo RRI para fins motivacionais dos professores para aplicarem as atividades numa aula.

b) Etapa 2 - Adaptar: fase intermediária com mudanças significativas - professores precisam ensinar processos de investigação científica com lições de pesquisa e resolução de problemas, o que requer duas ou mais aulas. Existe uma inserção casual de mais conteúdo RRI, mas sem propósito explícito.

c) Etapa 3 - Transformar: fase mais avançada com mudanças importantes - professores devem ensinar conteúdo científico com um tópico baseado em cenários para que os estudantes possam desenvolver seus projetos em três ou mais aulas. Existe uma inserção proposital com mais conteúdos e requer tempo para desenvolver habilidades de investigação científica para RRI. 


\section{REA}

Nos tempos atuais, com o crescimento da internet e a criação de diferentes mídias, a Educação tende a modernizar-se e a incorporar soluções que possam atender ao perfil dos estudantes. A cultura digital está cada vez mais sendo ampliada, pois existe uma perspectiva das populações mundiais e do Brasil de acesso à informação de forma simultânea, e mais, pois com o acesso à internet pelos dispositivos móveis, em todo tempo e em todos os lugares, não existem mais limites. As pessoas passaram de consumidores a produtores de conteúdo e é importante que a educação traga para os espaços educativos propostas que envolvam possibilidades de personalizar, aprimorar e redistribuir recursos capazes de melhorar a experiência dos estudantes e, consequentemente, ampliar a aprendizagem.

Percebe-se um movimento cada vez mais amplo no estudo e na divulgação dos REA (Recursos Educacionais Abertos) em todo o mundo, por significarem uma mudança de paradigma para a Educação e por também oferecerem possibilidade de desenvolver objetos de aprendizagens, recursos midiáticos ou mesmo ferramentas capazes de ampliar o acesso a um processo de ensino-aprendizagem de maior qualidade. Por ser um processo mundial é interessante verificar a definição mundial de REA, apesar de esta também estar em constante evolução e adequação, de acordo com as inovações legais e tecnológicas, como observa-se a seguir.

\section{Quadro 1 - REA}

Organização das Nações Unidas para a Educação, a Ciência e a Cultura (Unesco) em $2002^{1}$.
Considera-se um recurso educacional aberto qualquer tipo de ferramenta, material ou técnica de ensino e pesquisa, sustentado por uma mídia e que esteja registrado sob domínio público ou sob uma licença livre, podendo ser utilizado ou mesmo modificado por terceiros.

1 Disponívelem:<http://portal.unesco.org/ci/en/ev.phpURL_ID=2492\&URL_DO=DO_TOPIC\&URL_SECTION=201>. 


\begin{tabular}{|c|c|}
\hline $\begin{array}{l}\text { Definição de Recurso } \\
\text { Educacional Aberto (REA) } \\
\text { resultante do texto da } \\
\text { UNESCO/Common wealth } \\
\text { of Learning } 2011^{2} \text {. }\end{array}$ & $\begin{array}{l}\text { Podem ser materiais para serem utilizados no processo de } \\
\text { ensino-aprendizagem, na pesquisa, por meio de suportes } \\
\text { ou mídias diferenciadas, que estão sob a licença de domínio } \\
\text { público, ou aberta. O diferencial é que podem ser utilizados ou } \\
\text { adaptados por outras pessoas. }\end{array}$ \\
\hline $\begin{array}{l}\text { Declaração de Paris sobre } \\
\text { Recursos Educacionais } \\
\text { Abertos, } 2012^{3} \text {. }\end{array}$ & $\begin{array}{l}\text { REA são materiais de ensino, aprendizagem e investigação } \\
\text { em quaisquer suportes, digitais ou outros, que se situem no } \\
\text { domínio público ou que tenham sido divulgados sob licença } \\
\text { aberta que permite acesso, uso, adaptação e redistribuição } \\
\text { gratuitos por terceiros, mediante nenhuma restrição ou poucas } \\
\text { restrições. O licenciamento aberto é construído no âmbito da } \\
\text { estrutura existente dos direitos de propriedade intelectual, tais } \\
\text { como se encontram definidos por convenções internacionais } \\
\text { pertinentes, e respeita a autoria da obra. }\end{array}$ \\
\hline REA nas pesquisas atuais & $\begin{array}{l}\text { Podem ser: conteúdos em diferentes mídias ou tecnologias, } \\
\text { ferramentas tecnológicas e as licenças de propriedade } \\
\text { intelectual para promover a publicação aberta. } \\
\text { Para ser considerado um REA, o recurso precisa permitir quatro } \\
\text { liberdades mínimas classificadas de "4Rs": revisar, reusar, } \\
\text { remixar e redistribuir. }\end{array}$ \\
\hline
\end{tabular}

Fonte: As autoras, 2017.

Observa-se que a formatação técnica dos recursos abertos tende a possibilitar tanto o acesso, quanto o reuso e a adaptação dos mesmos, sendo que estes podem ser objetos de aprendizagem mais simples, complexos e até trechos de cursos, sistemas de avaliação diferenciados, aplicativos, ferramentas de edição e de construção de materiais, ou novas possibilidades que forem criadas, tendem a proporcionar uma melhor aquisição do conhecimento.

Por perceber-se que

Há uma inadequação cada vez mais ampla, profunda e grave entre saberes separados, fragmentados, compartilhados entre disciplinas, e, por outro lado, realidades ou problemas cada vez mais polidisciplinares,

2 UNESCO/COL. Guidelines for open educational resources (OER) in higher education, 2011. Disponível em: <https://www.col.org/about/governance/col-board-minutes-excerpts>.

3 Disponível em: <http://www.unesco.org/fileadmin/MULTIMEDIA/HQ/CI/WPFD2009/Portuguese_ Declaration.html>. 
transversais, multidimensionais, transnacionais, globais e planetários (MORIN, p. 13, 2009).

É fundamental que haja um movimento tanto acadêmico, como social e político para que haja uma quebra de paradigma e os REA podem ser um dos caminhos capazes de oferecer aos estudantes e aos docentes a melhoria do desempenho e a oferta de propostas didático-pedagógicas capazes de reduzir a inadequação do modelo tradicional, que tem suas matrizes estanques e sólidas. Apesar do movimento ter iniciado nos EUA, no Brasil cada vez mais torna-se economicamente interessante o uso dos REA, como soluções capazes de atingir um número cada vez maior de pessoas, por meio de recursos educacionais de qualidade, podendo auxiliar na aprendizagem, por esta se tornar mais prazerosa e interativa.

Os REA tendem a motivar os estudantes e auxiliar no seu processo de aprendizagem, como já fora exposto nesta reflexão, mas tem um outro elemento importante a ser considerado que é o docente, que passa também de consumidor a produtor, de reutilizador de objetos, de adequador de recursos, ou seja, um criador, autor, customizador, tornando-se assim incluído digitalmente e inserido no contexto histórico atual.

O Brasil necessita urgentemente de uma legislação mais atualizada e que permita a atualização do Projeto de Lei 1.513/11 ${ }^{4}$, que define o que é REA e estabelece obrigações do setor público nos momentos de aquisição de material didático digital ou físico, na qual é citada a possibilidade da produção intelectual que fora direta ou indiretamente financiada por recursos públicos ser livremente disponibilizada, com autorização expressa do seu autor, para utilização como REA. O Plano Nacional de Educação (PNE) busca em suas metas ampliar a qualidade da Educação e para tal a diversidade de propostas pedagógicas e a utilização de uma quantidade maior de recursos, tendem a dar suporte as atividades educacionais.

A colaboração entre os docentes e produtores de conteúdo é fundamental para o crescimento da utilização dos REA, e também uma

4 Disponível em: <http://www.camara.gov.br/proposicoesWeb/fichadetramitacao?idProposicao=505535>. 
construção diferenciada, definida como cocriação, ou seja, uma produção coletiva de um texto ou mídia, realizada por meio de relações interpessoais maduras entre pares ou grupos de autores em torno de um tema.

Acredita-se que cada vez mais os processos de construção coletiva tendem a crescer, principalmente quando inseridos em redes virtuais, pois possibilitam que haja trocas e aprendizagens variadas, como argumenta Torres (2002, p. 88) quando cita que os membros do grupo podem interagir com qualquer um dos colegas, estabelecendo uma rede de comunicação, sendo que cada um ou todos assumem os papéis de escritor, pesquisador, revisor e crítico.

É na gestão do processo de criação coletiva "que os componentes do grupo se organizam, repartem papéis, discutem ideias e posições, interagem entre si, definem sub-tarefas, tudo isso, dentro de uma proposta elaborada, definida e negociada coletivamente" (TORRES, 2002, p. 75).

Percebe-se ser fundamental que as instituições educacionais, presenciais ou virtuais, entendam os estudantes e educadores como efetivos protagonistas do processo de ensino-aprendizagem e os REA como uma opção interessante para dar suporte a esse tipo de prática pedagógica.

\section{Cocriação}

Nos estudos sobre REA e RRI tem um elemento que é fundamental para que ocorra a transformação esperada para educação, na qual estudantes, docentes e comunidade possam produzir conhecimento. Para tal a cocriação passa a ser um dos recursos interessante para esta mudança.

Entende-se cocriação como "Uma produção coletiva de um texto ou mídia, realizada por meio de relações interpessoais maduras entre pares ou grupos de autores em torno de um tema" (TORRES et al., 2010). Esta construção parte da troca constante entre os autores envolvidos no 
processo. No qual todos são responsáveis pelas ideias, criações, experiências, pesquisas e produções.

Corrobora com a pesquisadora Santos (2012, p. 15), "desenvolvimento de ações que estimulem a participação dos alunos, que valorizem a aprendizagem colaborativa, a pesquisa e o compartilhamento de diversas fontes de informações" são fundamentais para o processo de aprendizagem.

A seguir apresenta-se um processo de colaboração entre os pares que permitiu a cocriação em rede, a partir do dilema proposto pelo projeto Engage. O grupo de pesquisa PRAPETEC desafiou vinte docentes e dois alunos pesquisadores para realizarem as etapas de construção com estudantes do Ensino Médio e do Ensino Superior, sobre o tema: Agrobiodiversidade - Transgênicos, as atividades realizadas pelos participantes foram:

Quadro 2 - Atividades do Projeto

\begin{tabular}{|l|l|}
\hline Explorar os ambientes WeSpot e nQuire-it & $\begin{array}{l}\text { Exposição de fotos com compartilhamento } \\
\text { pelo Facebook }\end{array}$ \\
\hline Entrevistas com especialistas & $\begin{array}{l}\text { Exposição de pôsteres feitos por alunos e } \\
\text { compartilhados no Facebook }\end{array}$ \\
\hline Criação de mapas conceituais e mentais & Aulas expositivas \\
\hline Criação de vídeos instrucionais & Criação de ilustrações \\
\hline Criação de jogos educativos & Pesquisas direcionadas \\
\hline Sinalizar em LIBRAS os vocabulários relacionados \\
\hline
\end{tabular}

Fonte: PRAPETEC, 2015.

Nestas etapas pode-se praticar a interação e a colaboração [...] que a aprendizagem resulta num processo dinâmico de envolvimento, partilha e construção conjunta do novo conhecimento realizado pelos membros da comunidade (DIAS, 2001, p. 01).

Destaca-se a atividade exposição de fotos realizadas na PUCPR, foram adicionadas perguntas relacionadas a consciência do consumo relacionado aos produtos Transgênicos. 
Figura 1 - Registro da Exposição

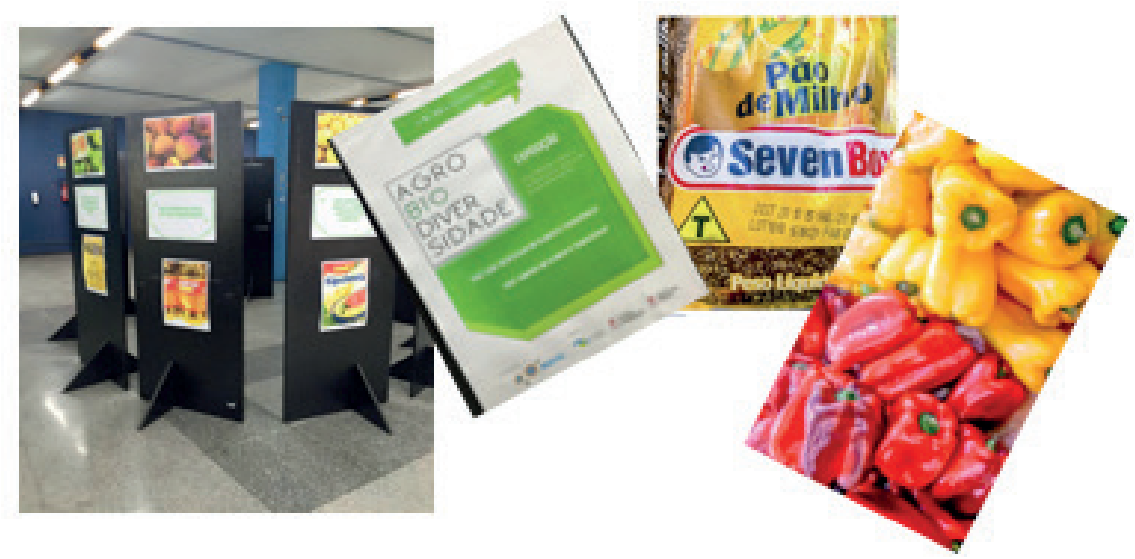

Fonte: PRAPETEC, 2016.

Sabe-se que ao oportunizar aos estudantes propostas inovadoras e que impliquem no compartilhamento em rede, é fundamental que ocorram os processos de cooperação e de colaboração. A pesquisadora Kenski (2008, p. 39-40) apresenta em seus estudos a importância das redes conectadas, mesmo que cada uma tenha suas características, pois: existe [...] "o momento da exposição, e este pode ser conectado com Recursos Educacionais Abertos e Redes Sociais: coaprendizagem e desenvolvimento profissional passado ou o futuro; seu espaço é reconfigurado a todo instante, de acordo com a perspectiva e os objetivos dos usuários".

A partir destas premissas, a exposição foi acompanhada em tempo real, na rede, por meio de uma página no Facebook que contou com postagens de vídeos e fotos da exposição. 


\section{Figura 2 - Facebook}

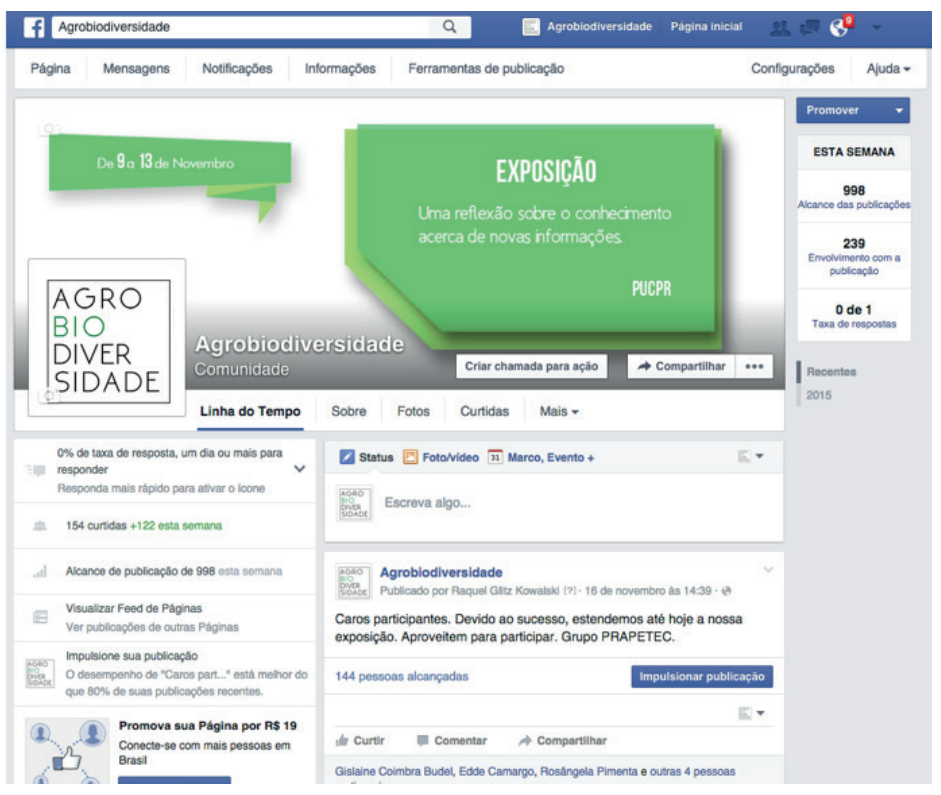

\begin{tabular}{|c|c|c|c|}
\hline \multicolumn{4}{|c|}{ Mostrando dados de 20/11/2015 - 26/11/2015 } \\
\hline Curtidas na Página & Alcance das publicaçōes & Envolvimento & > \\
\hline $\begin{array}{l}298 \text { Total de curtidas na Página } \\
\Delta 82.8 \% \text { desde a semana passada }\end{array}$ & $\begin{array}{l}1.666 \text { Alcance total } \\
\triangle 4.5 \% \text { desde a semana passada }\end{array}$ & 276 Pessoas envolvidas $i$ & \\
\hline 135 & 837 & 253 & \\
\hline $\begin{array}{l}\text { Novas curtidas na Página } \\
\Delta 6,3 \%\end{array}$ & $\begin{array}{l}\text { Alcance da publicaçalo } \\
\text {.0.4\% }\end{array}$ & Curtidas & \\
\hline $\begin{array}{l}\text { - Esta semana } \\
\text { - Semana passada }\end{array}$ & $\begin{array}{l}\text { - Esta semana } \\
\text { - Semana passada }\end{array}$ & $\begin{array}{l}4 \\
\text { Comentários }\end{array}$ & \\
\hline & & $\begin{array}{l}12 \\
\text { Compartilinamentos }\end{array}$ & \\
\hline $20 / 11 \quad 21 / 11 \quad 22 / 11 \quad 23 / 11 \quad 24 / 11 \quad 25 / 11 \quad 26 / 11$ & $20 / 11 \quad 21 / 11 \quad 22 / 11 \quad 23 / 11 \quad 24 / 11 \quad 25 / 11 \quad 26 / 11$ & Cliques em publicaçồ 6 & \\
\hline
\end{tabular}

Fonte: PRAPETEC, 2016. 
Percebe-se que deter a atenção dos estudantes, que estão cada vez mais conectados, exige posturas diferenciadas e recursos midiáticos diversos, um deles pode ser o YouTube, como na atividade da entrevista sobre Agrobiodiversidade - Transgênico, que contou com a participação de especialistas, e tinha como objetivo principal oferecer subsídio ao grupo PRAPETEC e demais participantes do projeto em rede. As entrevistas publicadas no YouTube sobre o tema ofereceram suporte teórico às discussões e escolhas no projeto.

Figura 3 - Entrevistadas

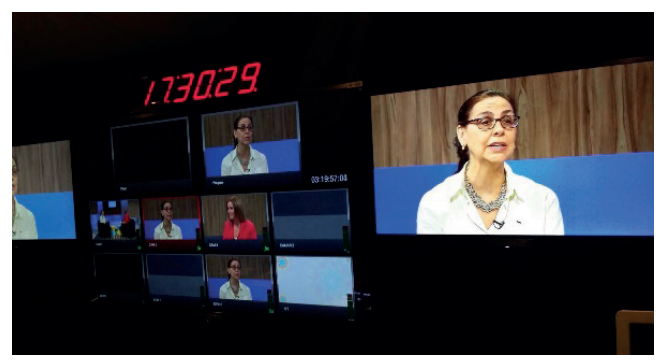

Fonte: Disponível em: <https://www.youtube.com/watch?v=RFrt7svfHbs>.

O projeto incluiu também, na ideia de educação híbrida, atividades presenciais com suporte nas propostas online, envolvendo docentes e estudantes do Ensino Médio e do Ensino Superior, com aulas expositivas e práticas sobre o tema em discussão. Nas atividades presenciais Agrobiodiversidade - Transgênicos foram envolvidos: 12 turmas na Educação Superior e 28 no Ensino Médio, 6 docentes e 583 estudantes.

Acredita-se que os estudantes de todas as áreas se beneficiam com propostas que proporcionam discussão, pesquisa e criação, sendo assim, o projeto envolveu o Curso Superior de Design Digital, especificamente a disciplina de Ilustração Aplicada, na qual realizaram a composição de imagens ilustradas. Neste momento, a imagem foi considerada como uma espécie de crônica autoral dos estudantes, que sintetizando o conteúdo, representaram graficamente suas ideias. 
Figura 4 - Produção dos estudantes na disciplina de Ilustração Aplicada

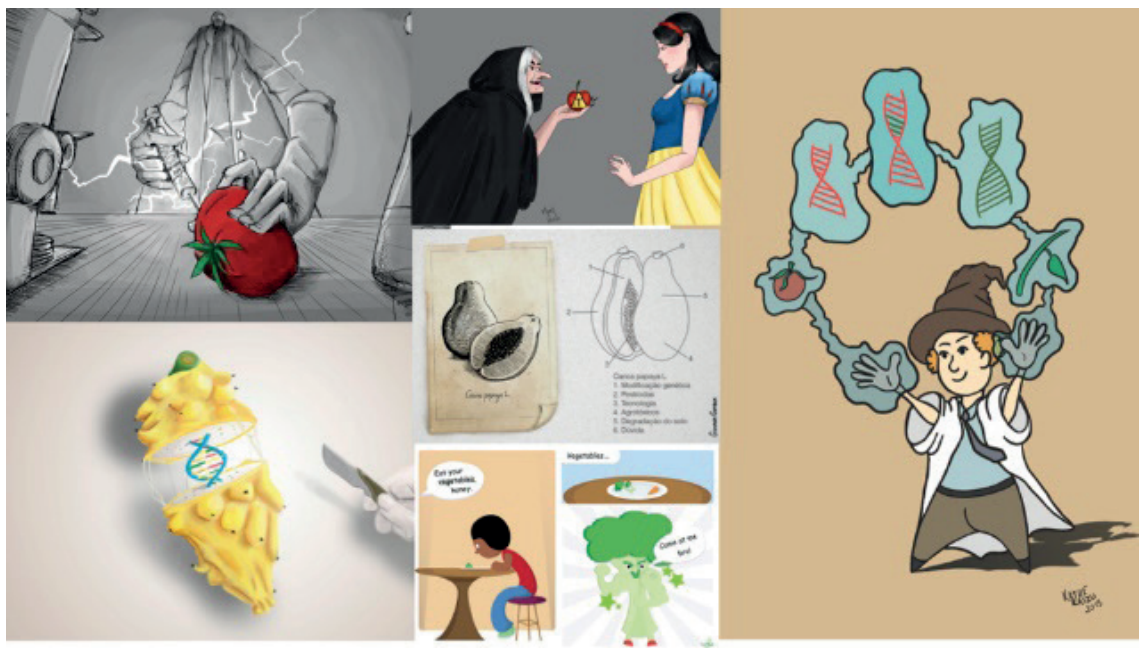

Fonte: PRAPETEC, 2016.

As artes criadas representaram ao mesmo tempo, a compreensão (ou não) dos estudantes/ilustradores referente ao tema dos alimentos transgênicos, e potencializaram o uso de imagens digitais ilustradas como interface na educação.

No mesmo curso, na disciplina de Ambientes Interativos, a proposta fora praticar a criação de recursos instrucionais, focando a criação de conteúdo online interativo. A atividade iniciou com a pesquisa sobre o tema Transgênicos e a criação posterior de um vídeo informando os conteúdos pesquisados. Num segundo momento, houve o desenvolvimento de revistas digitais, games e aplicativos interativos que foram postados no YouTube sob a licença CC. 
Figura 5 - Produção dos estudantes na disciplina de Ambientes Interativos
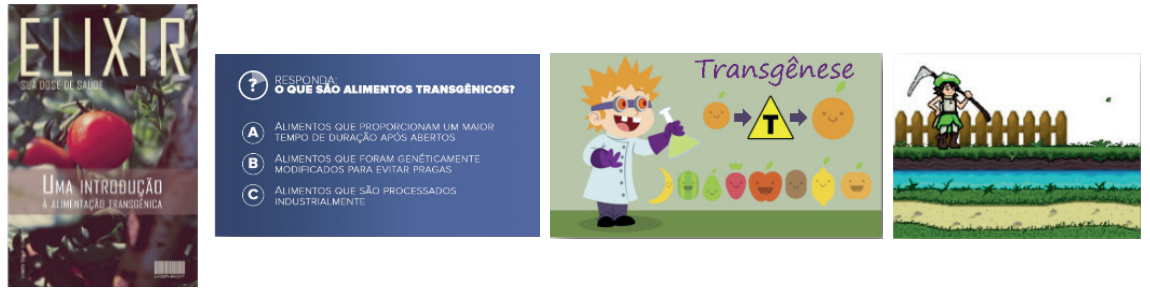

Fonte: PRAPETEC, 2016.

No Curso Superior de Design Digital, os estudantes foram desafiados a buscar sugestões para que as pessoas pudessem ter uma vida mais saudável. Na primeira etapa, tiveram que pesquisar sobre o tema e inserir suas considerações e resultados coletados, por meio de texto e fotos, nas redes sociais da disciplina, aproveitando para apresentar questões acerca da alimentação, dos alimentos e de sua origem.

Figura 6 - Produção dos estudantes: Curso Superior de Design Digital nas redes sociais

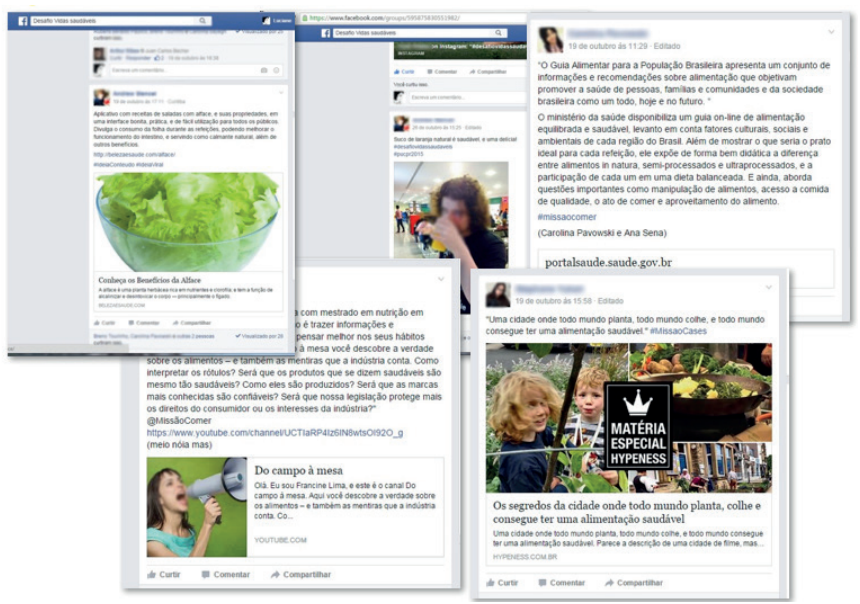

Fonte: PRAPETEC, 2016. 
Numa segunda etapa, discutiram em grupo acerca do levantado, fizeram mapas mentais das propostas e ideias e depois foram para os computadores desenvolver as soluções em formato de aplicativo.

Figura 7 - Produção dos estudantes: Curso Superior de Design Digital
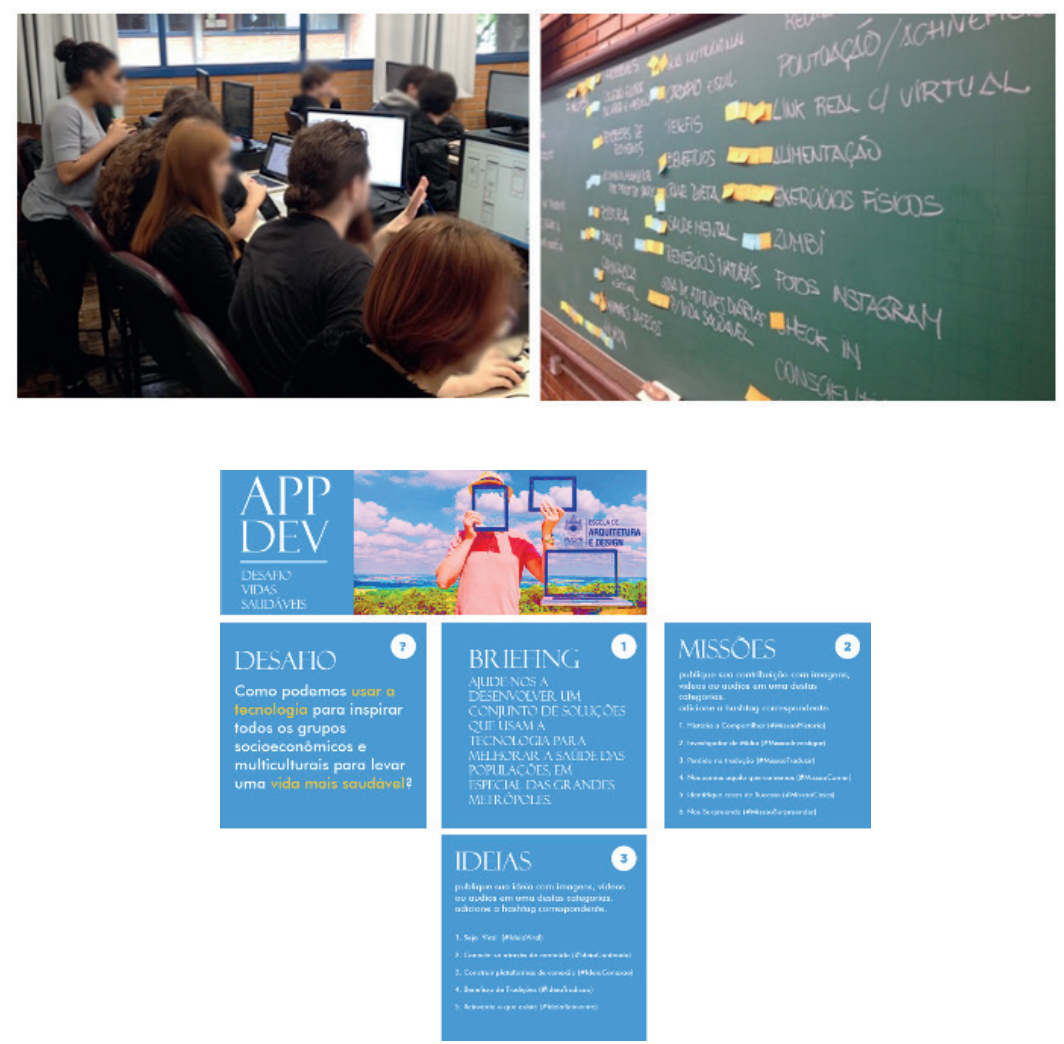

Fonte: PRAPETEC, 2016.

A LIBRAS, tem um papel cada vez maior na sociedade moderna, principalmente quando se reflete sobre futuros docentes e profissionais considerados como elementos de acessibilidades às informações para pessoas surdas. 
A docente responsável pela disciplina de LIBRAS das turmas dos cursos de Licenciaturas: Química; Física; Matemática; Educação Física; Ciências Sociais; Letras-Língua Portuguesa; Letras-Língua Inglesa; Letras-Língua Espanhola; Ciências Biológicas; História; Filosofia; Música, e também nos cursos de Psicologia; Gastronomia; Engenharia Elétrica; Jornalismo; Design Industrial, propôs envolver 350 estudantes, para desenvolverem as práticas pedagógicas, com fins de "sinalizar em LIBRAS os vocabulários relacionados aos TRANSGÊNICOS, contextualizando-os em situações comunicativas". Para tal, os alunos pesquisaram, elaboraram textos, utilizaram Alfabeto digital, realizaram o processo intertextual e interlingual e registraram as opiniões e sinalizações em vídeos e fotografias.

As etapas realizadas pelos estudantes por meio das vivências oportunizaram a percepção que é fundamental assegurou aos surdos o acesso às discussões sobre o tema, com interações comunicativas legitimando-os como sujeitos críticos e sociais na busca de soluções coletivas e conscientes.

Figura 8 - Estudantes de LIBRAS

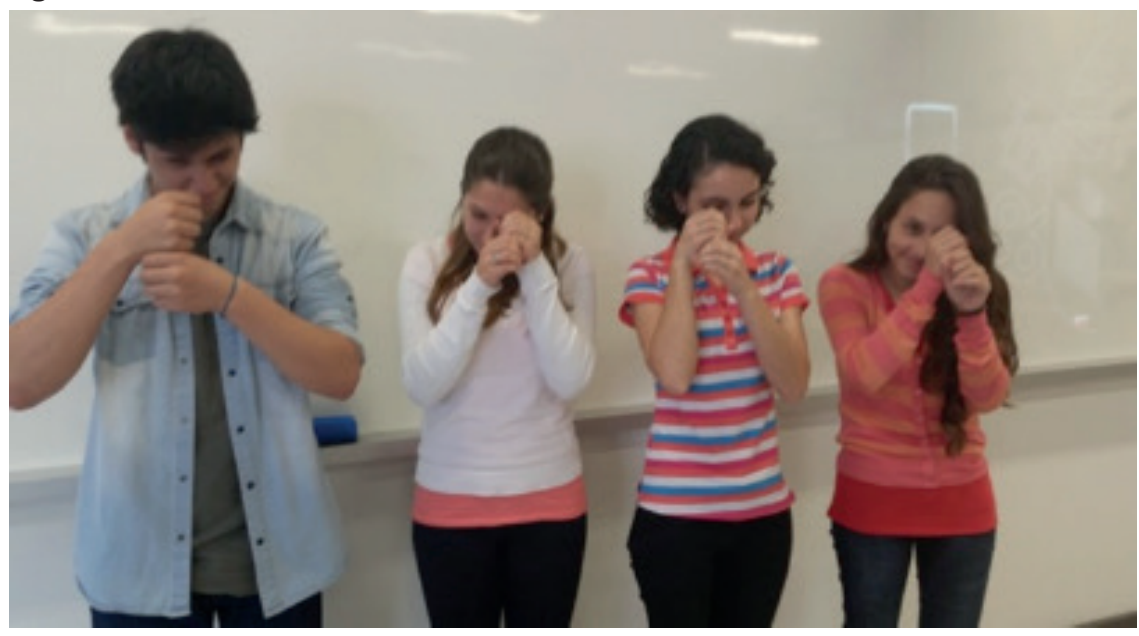

Fonte: PRAPETEC, 2016. 
O projeto foi realizado também no Curso de Pedagogia, nas turmas do $8^{\circ}$ período (Diurno e Noturno) em uma atividade desenvolvida em grupos de até cinco estudantes, sendo que cada grupo pesquisou a respeito do tema "Transgênicos" e em seguida, foi aberto o debate a respeito dos resultados encontrados. Como produto resultante da discussão, os grupos elaboraram jogos educativos e apresentaram aos demais colegas da turma, enfatizando como seria a proposta de utilização do mesmo em sala de aula. Os jogos elaborados foram: jogo da memória, dominó, jogo da velha, fecha a caixa, quebra-cabeça e jogos de percurso, todos com enfoque no tema Transgênicos.

Figura 9 - Jogos desenvolvidos pelos estudantes
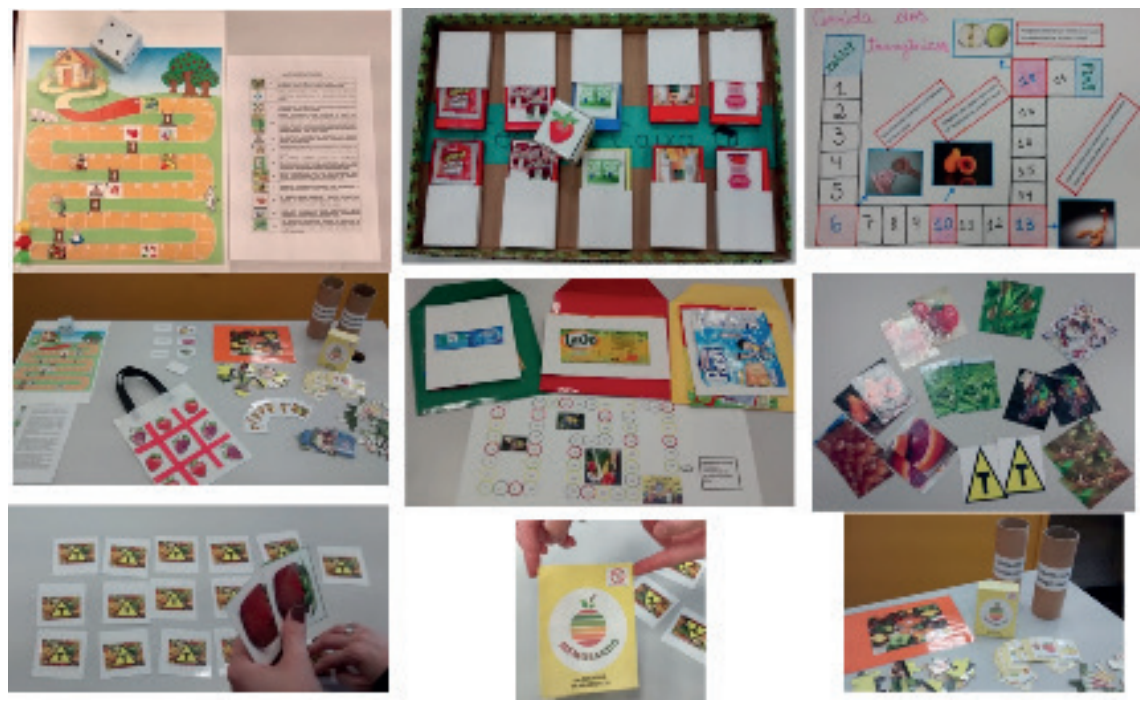

Fonte: PRAPETEC, 2016.

Os estudantes do Ensino Médio da rede pública estadual de ensino do Paraná, turmas do $1^{\mathrm{a}}$, $2^{\mathrm{a}}$ e $3^{\mathrm{a}}$ anos também foram envolvidos no projeto na pesquisa: "Sabemos o que comemos?" 
A fase inicial foi a consulta em fontes abertas na internet (artigos, vídeos, fotos), a busca de evidências na merenda escolar (transgênicos). Na segunda fase ocorreu a entrevista com profissionais da área da Biologia e Agronomia e, em seguida, foi feita a comunicação de informações em plenária e produção de breve mapa conceitual.

Figura 10 - Mapa conceitual
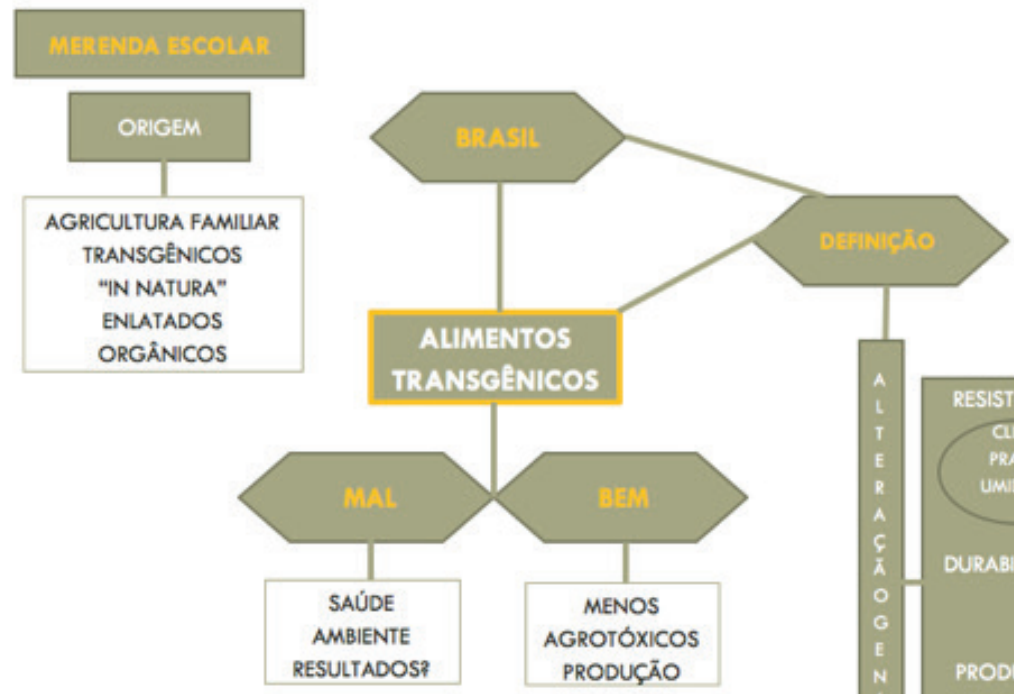

RESISTENCIA

CIIMA

PRAGA

UMIDADE

DURABILIDADE

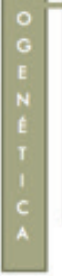

PRODUÇÃO

Fonte: PRAPETEC, 2016.

Na terceira fase ocorreu a divulgação interna por meio de exposição no colégio e elaboração de vídeo. 
Figura 11 - Exposição dos estudantes
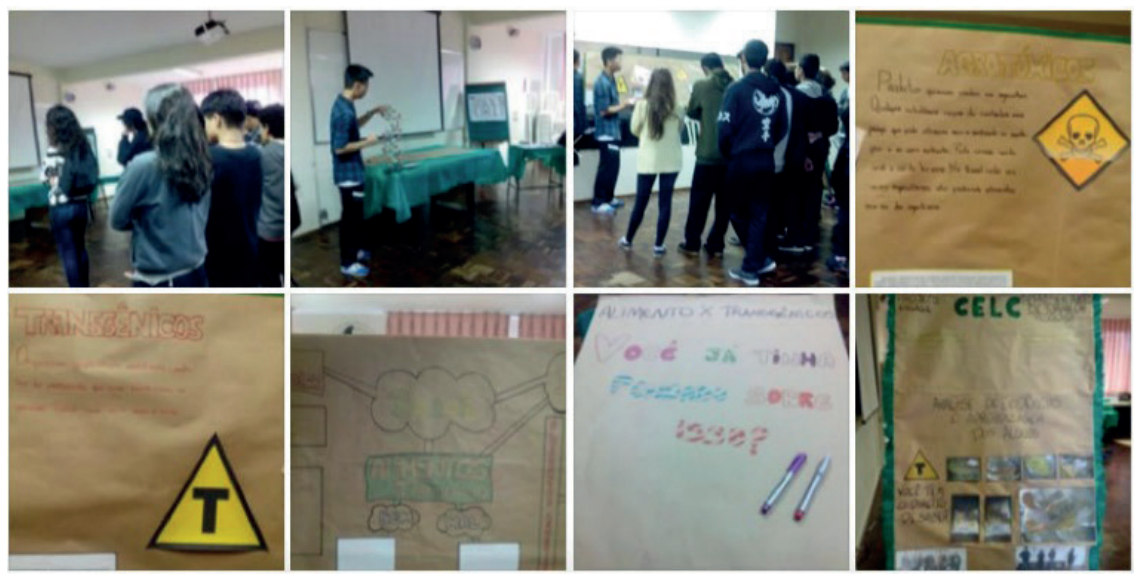

Fonte: PRAPETEC, 2016.

Os alunos interagiram com pesquisadores e co-criaram vários exemplos para comunicar seus resultados. Os usos das tecnologias favoreceram as discussões do grupo e as dez habilidades da RRI, exploradas em todas as atividades em diversos aspectos. Consegue-se perceber que a educação ambiental, contextualizada pelos próprios atores da sociedade que participaram das iniciativas, visam aumentar a conscientização do RRI no Brasil através de uma abordagem inclusiva, que vai além do objetivo do projeto Engage, incluindo universitários, pessoas surdas, escola primária e comunidades locais (OKADA et al, 2016).

\section{Considerações finais}

Os resultados apresentados nesta experiência são significativos e úteis para o projeto Engage e suas pesquisas na Europa e no Brasil. Embora existam diversos projetos na área de RRI financiados pela Comissão Europeia, como o ENGAGE (engagingscience.eu), muitas 
iniciativas são recentes e algumas questões ainda se encontram em aberto. Assim, faz-se necessário promover mais investigações, especialmente no que tange a resultados de aprendizagem e inclusão da abordagem na área de educação ambiental, de modo a promover a literacia digital científica (RATCLIFFE, 2003).

Pode-se afirmar que temas abrangentes, de cunho sócio ambiental, que influenciam a vida das pessoas diretamente são fundamentais para a sociedade. Os desafios postos para que professores europeus inovem no ensino, de maneira a promover habilidades para RRI, já foram apresentados em pesquisas anteriores (Kikis-Papadaskis \& Chaimala, 2015). A experiência aqui apresentada, que compôs uma das atividades de pesquisa do projeto ENGAGE, considera esses desafios, e pode ser referência para o uso de RRI na educação ambiental.

\section{Referências}

AIKENHEAD. Consequences to learning science through STS: A research perspective. In: SOLOMON, J.; AIKENHEAD, G. (Eds.), STS Education: International Perspective on Reform. New York: Teachers College Press, Columbia University, 1994. p. 169-186.

COMISSÃO EUROPEIA. Horizon 2020. The EU Framework Programme for Research and Innovation. Disponível em: <https://ec.europa.eu/programmes/horizon2020/ en/h2020-section/responsible-research-innovation>. Acessado em: 24 jul. 2017.

CONGRESSO MUNDIAL SOBRE RECURSOS EDUCACIONAIS ABERTOS (Rea) de 2012, Unesco, PARIS. Declaração de Paris sobre Recursos Educacionais Abertos, 2012. Disponível em: <http://www.unesco.org/fileadmin/MULTIMEDIA/HQ/ CI/WPFD2009/Portuguese_Declaration.html>. Acessado em: 10 de set. 2017.

DIAS, P. Comunidades de Conhecimento e Aprendizagem Colaborativa. In: Seminário Redes de Aprendizagem, Redes de Conhecimento, Conselho Nacional de Educação. Lisboa, 2001.

KENSKI, V. Tecnologias e ensino presencial e a distância. Campinas, SP: Papirus, 2008. 
KENSKI, V.; GOZZI, M.; JORDÃO, T. A experiência de ensinar e aprender em ambientes virtuais abertos. In: OKADA, A. (Ed.), 2012. Open Educational Resources and Social Networks: Co-Learning and Professional Development. London: Scholio Educational Research \& Publishing, 2012.

KENSKI, V. Novos processos de interação e comunicação no ensino mediado pelas tecnologias. Universidade de São Paulo. Faculdade de Educação - FEUSP. 2008.MORIN, E. A Cabeça bem feita: repensar a reforma, reformar o pensamento. Rio de Janeiro: Bertrand Brasil, 2002. Disponível em: < http://www.prpg.usp.br/ attachments/article/640/Caderno_7_PAE.pdf>. Acesso em: 03 set. 2017

OKADA, A.; YOUNG, G.; SHERBORNE, T. Innovative Teaching of Responsible Research and Innovation in Science Education. In: Open Education Europe, 2015. Disponível em: <https://www.openeducationeuropa.eu/en/article/InnovativeTeaching-of-Responsible-Research-and-Innovation-in-Science-Education $>$. Acesso em: 17 jul. 2017.

OKADA, A.; et al. Open Educational Resources for Responsible Research and Innovation: a case study with Brazilian universities and schools. In: International Conference on Responsible Research in Education and Management and its Impact, 2016. Disponível em: 〈http://www.engagingscience.eu/en/?wpdmdl=1761〉. Acesso em: 17 jul. 2017.

RATCLIFFE, M.; Grace, M. Science Education for Citizenship. Open University Press, 2003.

SANTOS, K. E. Aprendizagem colaborativa na educação a distância: Um caminho para a formação continuada. Dissertação (Mestrado em Educação) - Pontifícia Universidade Católica do Paraná, Curitiba, 2012.

TORRES, P. O Laboratório on-line de aprendizagem: uma proposta crítica de aprendizagem colaborativa para a educação. Tese (Doutorado do Programa de Pós-Graduação em Engenharia) - Florianópolis, Universidade Federal de Santa Catarina, 2002.

TORRES, P. L.; et al. Construção coletiva do conhecimento: desafios da cocriação no paradigma da complexidade. In: OKADA, A. (Ed.). Open Educational Resources and Social Networks: CoLearning and Professional Development. London: Scholio Educational Research \& Publishing, 2010. 
UNESCO/Commonwealth of Learning 2011, Recurso Educacional Aberto (REA). UNESCO/COL. Guidelines for open educational resources (OER) in higher education, 2011. Disponível em: <https://www.col.org/about/governance/col-board-minutes-excerpts $>$. Acesso em: 03 de set. 2017.

UNESCO. Organização das Nações Unidas para a Educação, a Ciência e a Cultura, 2002. Disponível em: <http://portal.unesco.org/ci/en/ev.phpURL_ID=2492\&URL_ DO=DO_TOPIC\&URL_SECTION=201.html >. Acesso em: 12 de set. 2017.

Recebido: 05/07/2017

Received: 07/05/2017

Recibido: 05/07/2017

Aprovado: 18/10/2017

Approved: 10/18/2017

Aprobado: 18/10/2017 